MATHEMATICS OF COMPUTATION

Volume 74, Number 251, Pages 1053-1065

S 0025-5718(04)01696-5

Article electronically published on September 10, 2004

\title{
CONVERGENCE \\ OF A STEP-DOUBLING GALERKIN METHOD FOR PARABOLIC PROBLEMS
}

\author{
BRUCE P. AYATI AND TODD F. DUPONT
}

\begin{abstract}
We analyze a single step method for solving second-order parabolic initial-boundary value problems. The method uses a step-doubling extrapolation scheme in time based on backward Euler and a Galerkin approximation in space. The technique is shown to be a second-order correct approximation in time. Since step-doubling can be used as a mechanism for step-size control, the analysis is done for variable time steps. The stability properties of step-doubling are contrasted with those of Crank-Nicolson, as well as those of more general extrapolated theta-weighted schemes. We provide an example computation that illustrates both the use of step-doubling for adaptive time step control and the application of step-doubling to a nonlinear system.
\end{abstract}

\section{INTRODUCTION}

In this paper we consider a numerical method for solving second-order parabolic initial-boundary value problems. This single step method consists of taking two half steps of backward Euler over a time interval to obtain one approximate solution and then a single step over that same interval to obtain another [8, 12. Having both approximations gives us two things: the two approximations can be compared to get an estimate of the size of the time-integration error; and the two firstorder correct approximations can be extrapolated to obtain a second-order correct approximate solution. The first of these benefits can be used to obtain a simple, yet effective, adaptive time-stepping algorithm. We use a Galerkin method for the spatial discretization.

The method can also be viewed as a rational approximation to the exponential (but not a Padé approximation [9]). Bramble and Sammon [3] showed convergence in the case of fixed time steps for a class of single step methods, including this method, that can be represented as rational approximations to the exponential.

We compare the stability properties of step-doubling with those of Crank-Nicolson for the case of a first-order linear ordinary differential equation, as well as those of more general extrapolated theta-weighted schemes.

Received by the editor October 22, 2003 and, in revised form, February 27, 2004.

2000 Mathematics Subject Classification. Primary 65M06, 65M12, 65M60; Secondary 35K15, $35 \mathrm{~K} 20,65 \mathrm{M} 15$.

Key words and phrases. Variable time steps, step-size control, parabolic partial differential equation.

The second author was supported by the ASCI Flash Center at the University of Chicago under DOE contract B532820, and by the MRSEC Program of the National Science Foundation under award DMR-0213745.

(C)2004 American Mathematical Society 
We analyze step-doubling using an energy method. Since one of the reasons to use step-doubling is step-size control, the analysis is done for variable time steps. In this paper, we present a particular step-size control strategy for step-doubling. A large number of our friends and collaborators have successfully used similar strategies 1, 4, 5, 6, 7, 10, 11]. We conjecture, based on watching our colleagues, that choosing a reasonable first guess at a tolerance based on a change in the solution is easier for most people than picking a bound for some second or third time derivative which would appear as a time truncation term that forces the differential equation. One feature of the analysis presented here is that the size of a time step is limited to less than three halves the size of the previous time step.

We provide an example computation that illustrates both the use of step-doubling for adaptive time step control and the application of step-doubling to a nonlinear system.

\section{THE PARABOLIC PROBLEM}

We consider a second-order linear parabolic partial differential equation with initial condition and homogeneous Dirichlet boundary condition on a bounded spatial domain $\Omega$ and a time interval $J=\left[t_{0}, t_{f}\right]$ :

$$
\begin{aligned}
\partial_{t} y-\nabla \cdot a(x) \nabla y+b(x, t) \cdot \nabla y & =f(x, t), & & x \in \Omega, t \in J, \\
y(x, t) & =0, & & x \in \partial \Omega, t \in J, \\
y\left(x, t_{0}\right) & =y_{0}(x), & & x \in \Omega,
\end{aligned}
$$

where $\nabla$ and $\nabla$. denote the gradient and divergence, respectively, in $x$. A weak form for this equation is that for every $v \in H_{0}^{1}(\Omega)$,

$$
\left(\partial_{t} y, v\right)+a(y, v)+b(y, v)=(f, v),
$$

where $(\cdot, \cdot)$ denotes the $L^{2}$ inner product, and the $a$ and $b$ denote the quadratic forms

$$
\begin{aligned}
& a(\varphi, \psi)=\int_{\Omega} a(x) \nabla \varphi \cdot \nabla \psi d x, \\
& b(\varphi, \psi)=\int_{\Omega}(b(x, t) \cdot \nabla \varphi) \psi d x .
\end{aligned}
$$

The distinction between the forms and the coefficients $a(x)$ and $b(x, t)$ should be clear from context. We also use the form $[a+b]$ defined by

$$
[a+b](\varphi, \psi)=a(\varphi, \psi)+b(\varphi, \psi) .
$$

We assume that the following conditions hold:

Condition 2.1. The functions $a(x)$ and $b(x, t)$ satisfy $a(x) \geq \underline{a}>0$ and $|b(x, t)| \leq \bar{b}$ for $x \in \Omega, t \in J$.

Condition 2.2. The form $[a+b]$ is nonsingular. 


\section{THE METHOD}

To solve (2.1)-(2.3), we use a step-doubling method based on a backward Euler method with local in time extrapolation. We consider a nonuniform time discretization, $t_{0}<t_{1}<\cdots<t_{m}=t_{f}$. We set $\Delta t_{n}=t_{n}-t_{n-1}$, for $n=1, \ldots, m$. Let $\varphi_{n}$ denote the value of $\varphi$ at time $t_{n}$ and $\varphi_{n-1 / 2}$ denote the value of $\varphi$ at time $\left(t_{n}+t_{n-1}\right) / 2$. We let $\mathcal{M}$ denote a finite dimensional subspace of $H_{0}^{1}(\Omega)$. We take $D_{n-1 / 2}, D_{n}, S_{n}, Y_{n-1}$ to be in $\mathcal{M}$. We solve the system

$$
\begin{aligned}
\left(\frac{D_{n-1 / 2}-Y_{n-1}}{\Delta t_{n} / 2}, v\right)+[a+b]\left(D_{n-1 / 2}, v\right) & =\left(f_{n-1 / 2}, v\right), \\
\left(\frac{D_{n}-D_{n-1 / 2}}{\Delta t_{n} / 2}, v\right)+[a+b]\left(D_{n}, v\right) & =\left(f_{n}, v\right), \\
\left(\frac{S_{n}-Y_{n-1}}{\Delta t_{n}}, v\right)+[a+b]\left(S_{n}, v\right) & =\left(f_{n}, v\right) .
\end{aligned}
$$

The computed solution at time $t_{n}$ is set to $Y_{n}=2 D_{n}-S_{n}$.

\section{Using STEP-DOUbLing TO CONTROL THE TIME STEP}

The idea of using step-doubling to estimate the time-integration error goes back at least to Gear [8], and Shampine [12] provides an overview of step-doubling methods as well as some issues with local extrapolation. In this section we provide a description of one of the ways that we have used step-doubling and remark on some of the aspects of its use that we like. There is a considerable degree of arbitrariness to the choices we make, and there are many other ways that one could deal with the details of using this method. The step-doubling process studied here provides two first-order correct approximations in time for each time step, $S_{n}$ and $D_{n}$. These can be compared to obtain a measure of the local time-truncation error. We choose a norm, $\|\cdot\|$, and a tolerance, tol. If $\left\|S_{n}-D_{n}\right\| \leq$ tol, we accept the results of the step and extrapolate to remove the first-order component of the error. If $\left\|S_{n}-D_{n}\right\|>$ tol, we reject the results of the step and recalculate using a smaller time step. In addition, we can adjust the next time step by the closeness of the error to the tolerance. The choice of the norm can depend strongly on what one feels is important about the solution.

An example scheme that tries to maintain the local time truncation error between $\frac{1}{4}$ tol and $\frac{3}{4}$ tol is given below. Take $\sigma=\sqrt[4]{2}$.

- If $\left\|S_{n}-D_{n}\right\|>$ tol, then we reject the step, and try again with a step size $\frac{\Delta t}{2}$.

- If $\left\|S_{n}-D_{n}\right\| \leq$ tol, then we accept the step and possibly adjust the next step according to the rules.

If $\left\|S_{n}-D_{n}\right\|>\frac{3 \text { tol }}{4}$, then $\Delta t=\Delta t / \sigma$.

If $\left\|S_{n}-D_{n}\right\|<\frac{\text { tol }}{4}$, then $\Delta t=\Delta t \sigma$, provided the last three steps were not rejected.

The choice of $\sigma=\sqrt[4]{2}$ is reasonable, but all that is needed is some number slightly greater than 1 . With the $\sigma$ given, if we are very far below the proper step size, $\Delta t$ will double every four steps or increase by a factor of 1024 in 40 steps. The reason for not increasing the step even if the error is far below the tolerance is that a rejected step indicates a dramatic change in the behavior of the solution; 
if we stepped across such a change and rejected a step we may well have a very small error indicator, but we have been warned there is roughness just ahead. In our experience this approach leads to a very small fraction of the time steps being rejected.

Two more remarks about step selection may be useful. If we have a target time $t_{\text {print }}$ that we want to be the end of a step, then we add a couple of rules to the step selection. When starting a step with a recommended time step that would take us past $t_{\text {print }}$, adjust the step to hit it exactly. When starting a step with a $\Delta t$ that will not make it to $t_{\text {print }}$, if $t_{\text {print }}$ is less than two such steps away, try to take a step with size half the distance to $t_{\text {print }}$. This pair of rules allows us to land at the given time without decreasing the size of the step by a large factor, something that is important given the moderate increases permitted by the rules. Of course, even these steps, where special rules were used to pick the $\Delta t$, can be rejected. The second situation that we have seen frequently is that the system is nonlinear and must be solved approximately. In this case the difficulty of solving the nonlinear system should be included in the choice to reject a step or to adjust the size of the step.

We note that the determination of tol for a particular application still requires a convergence study; this would be true even if we did not do the local extrapolation to try to remove the leading term in the error.

As mentioned in the Introduction, a number of our colleagues have used stepdoubling with time-stepping strategies similar to the one above 1 14, 5, 6] 7, 10, 11. We believe, based on this common experience, that most people will find that choosing a reasonable initial tolerance is easier than bounding second- and thirdorder derivatives in a time truncation term.

\section{A COMPARISON With CRANK-Nicolson AND EXTRAPOLATED THETA-WEIGHTED METHODS}

We consider the problem $y^{\prime}=A y$, where $A$ is a diagonalizable matrix. If $-a$ is an eigenvalue of $A$, we solve $y^{\prime}=-a y$ over a time step $\Delta t_{n}$ by step-doubling extrapolation to get

$$
Y_{n}=\left[2\left(\frac{1}{1+\frac{a \Delta t_{n}}{2}}\right)^{2}-\frac{1}{1+a \Delta t_{n}}\right] Y_{n-1},
$$

and by Crank-Nicolson to get

$$
Y_{n}=\left(\frac{2-a \Delta t_{n}}{2+a \Delta t_{n}}\right) Y_{n-1} .
$$

It should be noted that step-doubling involves three times the work of CrankNicolson. These rational approximations to $\exp \left(-a \Delta t_{n}\right)$ are compared in Figure 1 for $a \Delta t_{n} \in \mathbb{R}^{+}$. Note that the amplification factor for Crank-Nicolson tends to -1 as $a \Delta t$ gets large, so grossly under-resolved components bounce while slowly decaying.

We consider a theta-weighted scheme for the problem $y^{\prime}=-a y$. A single step of a theta-weighted scheme gives

$$
\frac{S_{n}-Y_{n-1}}{\Delta t_{n}}=-\theta a S_{n}+(\theta-1) a Y_{n-1}, \quad 0 \leq \theta \leq 1 .
$$




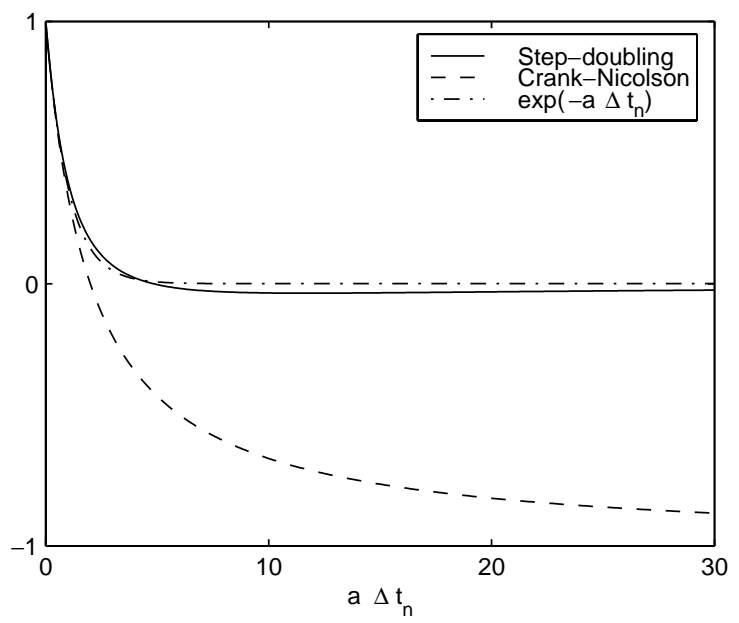

Figure 1. Amplification factors of step-doubling and CrankNicolson for the problem $y^{\prime}=-a y$.

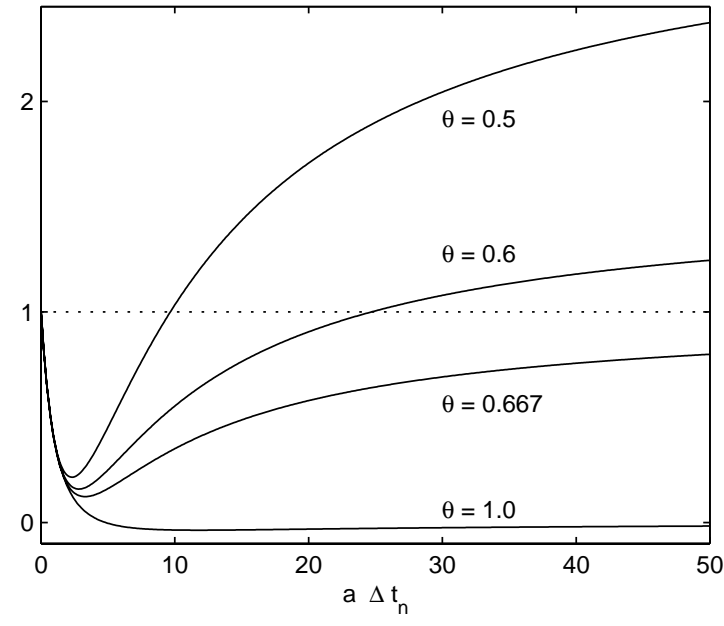

Figure 2. Amplification factors of step-doubling for various values of $\theta$ for a theta-weighted method.

If we take two half steps over this time interval to get an approximate solution $D_{n}$ and then extrapolate $Y_{n}=2 D_{n}-S_{n}$, we get a second-order correct approximation

$$
Y_{n}=\left[2\left(\frac{1+(\theta-1) \frac{a \Delta t_{n}}{2}}{1+\theta \frac{a \Delta t_{n}}{2}}\right)^{2}-\frac{1+(\theta-1) a \Delta t_{n}}{1+\theta a \Delta t_{n}}\right] Y_{n-1} .
$$

For $0 \leq \theta<\frac{2}{3}$, step-doubling is only conditionally stable. In particular, by taking $\theta=\frac{1}{2}$, step-doubling takes Crank-Nicolson, a stable second-order correct method, and yields only a conditionally stable second-order correct method. Figure 2 shows the amplification factors for various values of $\theta$. 


\section{Convergence}

In this section we state the main convergence result. The result is stated with some degree of generality with respect to the approximation space in space.

Let $\|\cdot\|$ and $\|\cdot\|_{H^{-1}}$ denote the $L^{2}$-norm and $H^{-1}$-norm, respectively, over $\Omega$. By $H^{-1}$ we mean the dual space of $H_{0}^{1}$. Let $\|\cdot\|_{A}$ denote the norm induced by $a(\cdot, \cdot)$. We denote by $L^{p}\left(t_{i}, t_{f} ; X\right)$ the $L^{p}$ norm on $\left(t_{i}, t_{f}\right)$ of the $X$-norm of a function.

Let $\partial_{t} y$ denote $\partial y / \partial t$ and $y_{n}$ denote $y\left(\cdot, t_{n}\right)$. Define, for $n=1, \ldots, m$,

$$
\begin{aligned}
d_{n-1 / 2} & =y_{n-1 / 2}+\frac{\left(\Delta t_{n}\right)^{2}}{8} \partial_{t}^{2} y_{n-1 / 2}, \\
d_{n} & =y_{n}+\frac{\left(\Delta t_{n}\right)^{2}}{4} \partial_{t}^{2} y_{n} .
\end{aligned}
$$

These functions will be used in the analysis because they are closer to the approximate solutions than the corresponding value of $y$ by one power of $\Delta t_{n}$.

We use elliptic projections in our convergence result [14]. Let $P$ be the projection with respect to the $[a+b]$ form where we choose $P \varphi=W \in \mathcal{M}$ such that, for every $v \in \mathcal{M},[a+b](\varphi-W, v)=0$. As is well known, $P y_{n}$ is a good approximation of $y_{n}$. Thus, bounding the difference between $Y_{n}$ and $P y_{n}$ is sufficient. We set

$$
\begin{aligned}
\eta_{n}=y_{n}-P y_{n}, & \vartheta_{n}=Y_{n}-P y_{n}, \\
\chi_{n}=d_{n}-P d_{n}, & \delta_{n}=D_{n}-P d_{n}, \\
\chi_{n-1 / 2}=d_{n-1 / 2}-P d_{n-1 / 2}, & \delta_{n-1 / 2}=D_{n-1 / 2}-P d_{n-1 / 2} .
\end{aligned}
$$

For a function of time $\varphi$, we define $(\Delta t)^{k} \varphi$ to be $\left(\Delta t_{n}\right)^{k} \varphi$ on $\left(t_{n-1} \cdot t_{n}\right)$.

Theorem 6.1. Assume $\Delta t_{n} \leq \frac{3-\xi}{2} \Delta t_{n-1}$ for $2 \leq n \leq m$, where $\xi$ is an arbitrary positive constant less than one. There exist positive constants $\Delta t_{c}$ and $C$, dependent only on $\underline{a}, \bar{b}$, and $\xi$, such that if $\Delta t_{n}<\Delta t_{c}$ for $1 \leq n \leq m$, then

$$
\begin{aligned}
& \left\|\vartheta_{m}\right\|^{2} \leq C\left(\left\|\vartheta_{0}\right\|^{2}+\Delta t_{1}\left\|\vartheta_{0}\right\|_{A}^{2}\right. \\
& \left.+\left\|(I-P) \partial_{t} y\right\|_{L^{2}\left(0, t_{m} ; H^{-1}\right)}^{2}+\left\|\Delta t(I-P) \partial_{t}^{2} y\right\|_{L^{2}\left(0, t_{m} ; H^{-1}\right)}^{2}\right) \\
& \left.\quad+\left\|(\Delta t)^{2} \partial_{t}^{2} y\right\|_{L^{2}\left(0, t_{m} ; H^{1}\right)}^{2}+\left\|(\Delta t)^{2} \partial_{t}^{3} y\right\|_{L^{2}\left(0, t_{m} ; H^{-1}\right)}^{2}\right) .
\end{aligned}
$$

\section{Proof of the CONVERGENCE RESUlt}

For the homogeneous case of equations 2.1 - 2.3 with $b=0$, with constant time steps and without spatial discretization, this method can be analyzed by eigenvalue expansion methods (see Section 5). For the generality desired in this paper, particularly the use of variable time steps, we show second-order convergence in time by an energy analysis 13 .

Let $C^{*}$ denote an arbitrary positive constant with dependencies at most on $\underline{a}$ and $\bar{b}$. Let $C_{\xi}$ denote an arbitrary positive constant with dependencies at most on $\underline{a}, \bar{b}$ and $\xi$. Let $K=\bar{b} / \sqrt{a}$.

Adding equations (3.1) and (3.2) and subtracting equation (3.3), recalling that $Y_{n}=2 D_{n}-S_{n}$, gives

$$
\left(\frac{Y_{n}-Y_{n-1}}{\Delta t_{n}}, v\right)+[a+b]\left(Y_{n}, v\right)+[a+b]\left(D_{n-1 / 2}-D_{n}, v\right)=\left(f_{n-1 / 2}, v\right) .
$$


For the true solution, we consider the variational form equation (2.4) at time $t_{n-1 / 2}$ to get

$$
\begin{aligned}
\left(\frac{y_{n}-y_{n-1}}{\Delta t_{n}}, v\right)+[a+b]\left(y_{n}, v\right)+[a+b]\left(y_{n-1 / 2}-y_{n}, v\right) & \\
& =\left(f_{n-1 / 2}, v\right)+\left(\rho_{n}, v\right)
\end{aligned}
$$

where

$$
\rho_{n}=\frac{y_{n}-y_{n-1}}{\Delta t_{n}}-\partial_{t} y_{n-1 / 2}
$$

Subtracting equation (7.2) from (7.1) gives

$$
\left(\frac{\vartheta_{n}-\vartheta_{n-1}}{\Delta t_{n}}, v\right)+a\left(\vartheta_{n}, v\right)+a\left(\delta_{n-1 / 2}-\delta_{n}, v\right)=G_{n}(v),
$$

where

$$
G_{n}(v)=\left(\frac{\eta_{n}-\eta_{n-1}}{\Delta t_{n}}-\rho_{n}, v\right)-b\left(\vartheta_{n}, v\right)-b\left(\delta_{n-1 / 2}-\delta_{n}, v\right) .
$$

We take $v=\vartheta_{n}$ to get

$$
\begin{aligned}
\frac{1}{2 \Delta t_{n}}\left(\left\|\vartheta_{n}\right\|^{2}-\left\|\vartheta_{n-1}\right\|^{2}+\left\|\vartheta_{n}-\vartheta_{n-1}\right\|^{2}\right)+ & \left\|\vartheta_{n}\right\|_{A}^{2} \\
& +a\left(\delta_{n-1 / 2}-\delta_{n}, \vartheta_{n}\right)=G_{n}\left(\vartheta_{n}\right) .
\end{aligned}
$$

Since $\frac{1}{4}\left\|\vartheta_{n}\right\|_{A}^{2}+a\left(\delta_{n}-\delta_{n-1 / 2}, \vartheta_{n}\right)+\left\|\delta_{n}-\delta_{n-1 / 2}\right\|_{A}^{2}=\left\|\vartheta_{n} / 2+\delta_{n}-\delta_{n-1 / 2}\right\|_{A}^{2}$, we have

$$
\begin{aligned}
& \frac{1}{2 \Delta t_{n}}\left(\left\|\vartheta_{n}\right\|^{2}-\left\|\vartheta_{n-1}\right\|^{2}+\left\|\vartheta_{n}-\vartheta_{n-1}\right\|^{2}\right)+\frac{3}{4}\left\|\vartheta_{n}\right\|_{A}^{2} \\
& +\left\|\vartheta_{n} / 2+\delta_{n}-\delta_{n-1 / 2}\right\|_{A}^{2}-\left\|\delta_{n}-\delta_{n-1 / 2}\right\|_{A}^{2}=G_{n}\left(\vartheta_{n}\right) .
\end{aligned}
$$

It is apparent that we need to control $\delta_{n}-\delta_{n-1 / 2}$. We can manipulate equation (2.4) to obtain

$$
\begin{aligned}
& \left(\frac{d_{n-1 / 2}-y_{n-1}}{\Delta t_{n} / 2}, v\right)+[a+b]\left(d_{n-1 / 2}, v\right) \\
& =\left(f_{n-1 / 2}, v\right)+\left(\mu_{n-1 / 2}, v\right)+\frac{\left(\Delta t_{n}\right)^{2}}{8}[a+b]\left(\partial_{t}^{2} y_{n-1 / 2}, v\right), \\
& \left(\frac{d_{n}-d_{n-1 / 2}}{\Delta t_{n} / 2}, v\right)+[a+b]\left(d_{n}, v\right) \\
& =\left(f_{n}, v\right)+\left(\mu_{n}, v\right)+\frac{\left(\Delta t_{n}\right)^{2}}{4}[a+b]\left(\partial_{t}^{2} y_{n}, v\right),
\end{aligned}
$$

where the time truncation terms are

$$
\begin{aligned}
\mu_{n-1 / 2} & =\frac{d_{n-1 / 2}-y_{n-1}}{\Delta t_{n} / 2}-\partial_{t} y_{n-1 / 2}, \\
\mu_{n} & =\frac{d_{n}-d_{n-1 / 2}}{\Delta t_{n} / 2}-\partial_{t} y_{n} .
\end{aligned}
$$

The terms $\mu_{n-1 / 2}$ and $\mu_{n}$ will be seen to be of second order in time. 
Subtracting equation (7.4) from (3.1) and equation (17.5) from (3.2) gives

$$
\begin{gathered}
\left(\frac{\delta_{n-1 / 2}-\vartheta_{n-1}}{\Delta t_{n} / 2}, v\right)+a\left(\delta_{n-1 / 2}, v\right)=R_{n}(v), \\
\left(\frac{\delta_{n}-\delta_{n-1 / 2}}{\Delta t_{n} / 2}, v\right)+a\left(\delta_{n}, v\right)=Q_{n}(v)
\end{gathered}
$$

where the operators

$$
\begin{aligned}
R_{n}(v)= & \left(\frac{\chi_{n-1 / 2}-\eta_{n-1}}{\Delta t_{n} / 2}-\mu_{n-1 / 2}, v\right) \\
& -\frac{\left(\Delta t_{n}\right)^{2}}{8}[a+b]\left(\partial_{t}^{2} y_{n-1 / 2}, v\right)-b\left(\delta_{n-1 / 2}, v\right), \\
Q_{n}(v)= & \left(\frac{\chi_{n}-\chi_{n-1 / 2}}{\Delta t_{n} / 2}-\mu_{n}, v\right)-\frac{\left(\Delta t_{n}\right)^{2}}{4}[a+b]\left(\partial_{t}^{2} y_{n}, v\right)-b\left(\delta_{n}, v\right) .
\end{aligned}
$$

If we set $v=\delta_{n-1 / 2}-\vartheta_{n-1}$ in equation (7.6) and $v=\delta_{n}-\delta_{n-1 / 2}$ in equation (7.7), we obtain

$$
\begin{aligned}
\frac{2}{\Delta t_{n}}\left\|\delta_{n-1 / 2}-\vartheta_{n-1}\right\|^{2}+\frac{1}{2}\left(\left\|\delta_{n-1 / 2}\right\|_{A}^{2}-\left\|\vartheta_{n-1}\right\|_{A}^{2}+\right. & \left.\left\|\delta_{n-1 / 2}-\vartheta_{n-1}\right\|_{A}^{2}\right) \\
& =R_{n}\left(\delta_{n-1 / 2}-\vartheta_{n-1}\right) \\
\frac{2}{\Delta t_{n}}\left\|\delta_{n}-\delta_{n-1 / 2}\right\|^{2}+\frac{1}{2}\left(\left\|\delta_{n}\right\|_{A}^{2}-\left\|\delta_{n-1 / 2}\right\|_{A}^{2}+\| \delta_{n}-\right. & \left.\delta_{n-1 / 2} \|_{A}^{2}\right) \\
& =Q_{n}\left(\delta_{n}-\delta_{n-1 / 2}\right) .
\end{aligned}
$$

Subtracting equation (7.6) from equation (7.7), with $v=\delta_{n}-\delta_{n-1 / 2}$, gives

$$
\begin{array}{r}
\frac{1}{\Delta t_{n}}\left(\left\|\delta_{n}-\delta_{n-1 / 2}\right\|^{2}-\left\|\delta_{n-1 / 2}-\vartheta_{n-1}\right\|^{2}+\left\|\delta_{n}-2 \delta_{n-1 / 2}+\vartheta_{n-1}\right\|^{2}\right) \\
+\left\|\delta_{n}-\delta_{n-1 / 2}\right\|_{A}^{2}=Q_{n}\left(\delta_{n}-\delta_{n-1 / 2}\right)-R_{n}\left(\delta_{n}-\delta_{n-1 / 2}\right) .
\end{array}
$$

Adding equations (7.8)-(7.10) gives

$$
\begin{gathered}
\frac{1}{\Delta t_{n}}\left(3\left\|\delta_{n}-\delta_{n-1 / 2}\right\|^{2}+3\left\|\delta_{n-1 / 2}-\vartheta_{n-1}\right\|^{2}+\left\|\delta_{n}-2 \delta_{n-1 / 2}+\vartheta_{n-1}\right\|^{2}\right) \\
+\frac{1}{2}\left(\left\|\delta_{n}\right\|_{A}^{2}-\left\|\vartheta_{n-1}\right\|_{A}^{2}+\left\|\delta_{n-1 / 2}-\vartheta_{n-1}\right\|_{A}^{2}+3\left\|\delta_{n}-\delta_{n-1 / 2}\right\|_{A}^{2}\right) \\
=R_{n}\left(\delta_{n-1 / 2}-\vartheta_{n-1}\right)+2 Q_{n}\left(\delta_{n}-\delta_{n-1 / 2}\right)-R_{n}\left(\delta_{n}-\delta_{n-1 / 2}\right),
\end{gathered}
$$

Equation (7.11) provides the desired control of $\delta_{n}-\delta_{n-1 / 2}$. We note that $\left\|\delta_{n}\right\|_{A}^{2}+$ $\left\|\delta_{n}-\delta_{n-1 / 2}\right\|_{A}^{2}-\frac{1}{2}\left\|\delta_{n-1 / 2}\right\|_{A}^{2}=2\left\|\delta_{n}-\frac{1}{2} \delta_{n-1 / 2}\right\|_{A}^{2} \geq 0$. Then adding equation (7.11) to equation (7.3) gives

$$
\begin{gathered}
\frac{1}{\Delta t_{n}}\left(\left\|\vartheta_{n}\right\|^{2}-\left\|\vartheta_{n-1}\right\|^{2}+6\left\|\delta_{n-1 / 2}-\delta_{n}\right\|^{2}+2\left\|\delta_{n-1 / 2}-\vartheta_{n-1}\right\|^{2}\right) \\
+\frac{1}{4}\left(3\left\|\vartheta_{n}\right\|_{A}^{2}-2\left\|\vartheta_{n-1}\right\|_{A}^{2}+\left\|\delta_{n}-\delta_{n-1 / 2}\right\|_{A}^{2}\right. \\
\left.+\left\|\delta_{n}\right\|_{A}^{2}+\frac{1}{2}\left\|\delta_{n-1 / 2}\right\|_{A}^{2}+2\left\|\delta_{n-1 / 2}-\vartheta_{n-1}\right\|_{A}^{2}\right) \\
=G_{n}\left(\vartheta_{n}\right)+R_{n}\left(\delta_{n-1 / 2}-\vartheta_{n-1}\right)+2 Q_{n}\left(\delta_{n}-\delta_{n-1 / 2}\right)-R_{n}\left(\delta_{n}-\delta_{n-1 / 2}\right) .
\end{gathered}
$$


We now require bounds on the right-hand side of equation (7.12). We note that $[a+b](w, z) \leq\|w\|_{A}\|z\|_{A}+K\|w\|_{A}\|z\|$, where we recall that $K=\bar{b} / \sqrt{\underline{a}}$. Using Hölder's Inequality and the inequality $\|v\|_{H^{1}} \leq(1 / \sqrt{\underline{a}})\|v\|_{A}+\|v\|$, we obtain the following bounds on the operators:

$$
\begin{aligned}
G_{n}(v) \leq & \left(\left\|\frac{\eta_{n}-\eta_{n-1}}{\Delta t_{n}}\right\|_{H^{-1}}+\left\|\rho_{n}\right\|_{H^{-1}}\right)\left(\frac{1}{\sqrt{\underline{a}}}\|v\|_{A}+\|v\|\right) \\
& +K\left\|\vartheta_{n}\right\|_{A}\|v\|+K\left\|\left(\delta_{n-1 / 2}-\delta_{n}\right)\right\|_{A}\|v\|, \\
R_{n}(v) \leq & \left(\left\|\frac{\chi_{n-1 / 2}-\eta_{n-1}}{\Delta t_{n} / 2}\right\|_{H^{-1}}+\left\|\mu_{n-1 / 2}\right\|_{H^{-1}}\right)\left(\frac{1}{\sqrt{\underline{a}}}\|v\|_{A}+\|v\|\right) \\
& +K\left\|\delta_{n-1 / 2}\right\|_{A}\|v\| \\
& +\frac{\left(\Delta t_{n}\right)^{2}}{8}\left(\left\|\partial_{t}^{2} y_{n-1 / 2}\right\|_{A}\|v\|_{A}+K\left\|\partial_{t}^{2} y_{n-1 / 2}\right\|_{A}\|v\|\right), \\
Q_{n}(v) \leq & \left(\left\|\frac{\chi_{n}-\chi_{n-1 / 2}}{\Delta t_{n} / 2}\right\|_{H^{-1}}+\left\|\mu_{n}\right\|_{H^{-1}}\right)\left(\frac{1}{\sqrt{\underline{a}}}\|v\|_{A}+\|v\|\right)+K\left\|\delta_{n}\right\|_{A}\|v\| \\
& +\frac{\left(\Delta t_{n}\right)^{2}}{4}\left(\left\|\partial_{t}^{2} y_{n}\right\|_{A}\|v\|_{A}+K\left\|\partial_{t}^{2} y_{n}\right\|_{A}\|v\|\right) .
\end{aligned}
$$

Let $\xi$ denote an arbitrary positive constant that is less than one. We use the above bounds on $G_{n}, R_{n}$, and $Q_{n}$, and assume $\Delta t_{n}$ is sufficiently small. Then using the arithmetic-geometric mean inequality, $w z \leq \varepsilon w^{2} / 2+z^{2} /(2 \varepsilon)$, with the appropriate choices of $\varepsilon$, we obtain

$$
\begin{aligned}
G_{n}\left(\vartheta_{n-1}\right) & +R_{n}\left(\delta_{n-1 / 2}-\vartheta_{n-1}\right)+2 Q_{n}\left(\delta_{n}-\delta_{n-1 / 2}\right)-R_{n}\left(\delta_{n}-\delta_{n-1 / 2}\right) \\
\leq & C^{*}\left(\left\|\frac{\eta_{n}-\eta_{n-1}}{\Delta t_{n}}\right\|_{H^{-1}}^{2}+\left\|\frac{\chi_{n-1 / 2}-\eta_{n-1}}{\Delta t_{n} / 2}\right\|_{H^{-1}}^{2}+\left\|\frac{\chi_{n}-\chi_{n-1 / 2}}{\Delta t_{n} / 2}\right\|_{H^{-1}}^{2}\right) \\
& +C^{*}\left(\left\|\rho_{n}\right\|_{H^{-1}}^{2}+\left\|\mu_{n-1 / 2}\right\|_{H^{-1}}^{2}+\left\|\mu_{n}\right\|_{H^{-1}}^{2}\right) \\
& +C^{*}\left(\Delta t_{n}\right)^{4}\left(\left\|\partial_{t}^{2} y_{n-1 / 2}\right\|_{A}^{2}+\left\|\partial_{t}^{2} y_{n}\right\|_{A}^{2}\right)+\frac{\xi}{4}\left\|\vartheta_{n-1}\right\|_{A}^{2}+C_{\xi}\left\|\vartheta_{n-1}\right\|^{2} \\
& +\frac{1}{\Delta t_{n}}\left(6\left\|\delta_{n-1 / 2}-\delta_{n}\right\|^{2}+2\left\|\delta_{n-1 / 2}-\vartheta_{n-1}\right\|^{2}\right) \\
& +\frac{1}{4}\left(\left\|\delta_{n}-\delta_{n-1 / 2}\right\|_{A}^{2}+\left\|\delta_{n}\right\|_{A}^{2}+\frac{1}{2}\left\|\delta_{n-1 / 2}\right\|_{A}^{2}+2\left\|\delta_{n-1 / 2}-\vartheta_{n-1}\right\|_{A}^{2}\right) .
\end{aligned}
$$

We recall that $C^{*}$ is an arbitrary constant with dependencies on at most $\underline{a}$ and $\bar{b}$ and $C_{\xi}$ with dependencies on at most $\underline{a}, \bar{b}$ and $\xi$.

We now need bounds on the $H^{-1}$-norms of $\rho_{n}, \mu_{n-1 / 2}$, and $\mu_{n}$. Manipulation of Taylor series gives the following equations for the errors due to approximating the time derivatives (see [2] for details):

$$
\begin{aligned}
\rho_{n} & =\frac{1}{\Delta t_{n}}\left(\int_{t_{n-1}}^{t_{n-1 / 2}} \frac{\left(t_{n-1}-t\right)^{2}}{2} \partial_{t}^{3} y(x, t) d t+\int_{t_{n-1 / 2}}^{t_{n}} \frac{\left(t_{n}-t\right)^{2}}{2} \partial_{t}^{3} y(x, t) d t\right), \\
\mu_{n-1 / 2} & =\frac{1}{\Delta t_{n}} \int_{t_{n-1}}^{t_{n-1 / 2}}\left(t_{n-1 / 2}-t\right)^{2} \partial_{t}^{3} y(x, t) d t, \\
\mu_{n} & =\frac{\Delta t_{n}}{4}\left(\partial_{t}^{2} y_{n}-\partial_{t}^{2} y_{n-1 / 2}\right)+\frac{1}{\Delta t_{n}} \int_{t_{n-1 / 2}}^{t_{n}}\left(t_{n-1 / 2}-t\right)^{2} \partial_{t}^{3} y(x, t) d t .
\end{aligned}
$$


Note that for a function $\varphi \in H^{1}(z, w)$, we have the inequality $\varphi(w)-\varphi(z)=$ $\int_{z}^{w} 1 \cdot \varphi^{\prime}(s) d s \leq \sqrt{|w-z|}\left\|\varphi^{\prime}\right\|_{L^{2}([z, w])}$. We then get the following bounds:

$$
\begin{aligned}
\left\|\rho_{n}\right\|_{H^{-1}} & \leq \frac{\left(\Delta t_{n}\right)^{3 / 2}}{4}\left\|\partial_{t}^{3} y\right\|_{L^{2}\left(H^{-1} ;\left[t_{n-1}, t_{n}\right]\right)}, \\
\left\|\mu_{n-1 / 2}\right\|_{H^{-1}} & \leq \frac{\left(\Delta t_{n}\right)^{3 / 2}}{4}\left\|\partial_{t}^{3} y\right\|_{L^{2}\left(H^{-1} ;\left[t_{n-1}, t_{n}\right]\right)}, \\
\left\|\mu_{n}\right\|_{H^{-1}} & \leq \frac{\left(\Delta t_{n}\right)^{3 / 2}}{2}\left\|\partial_{t}^{3} y\right\|_{L^{2}\left(H^{-1} ;\left[t_{n-1}, t_{n}\right]\right)} .
\end{aligned}
$$

Using the bounds (7.13)-(77.16) and applying a discrete Gronwall's Inequality 1 to equation (7.12) gives Theorem 6.1

\section{An EXAMPLE SYSTEM}

We present an example system that illustrates the utility of step-doubling extrapolation for the adaptive time integration of parabolic problems. Although the parabolic system considered in the analysis is linear, we choose an example system that is nonlinear in space to show the applicability of step-doubling to nonlinear systems.

The approximate solutions computed in this section were obtained using the freely available software toolkit BuGS [1]. BuGS is a toolkit for solving single space dimensional, nonlinear systems of partial differential equations which are at most order one in time and order two in space. The user defines the spatial discretization of the equations by writing a residual function based on first-order backward differences in time. BuGS then uses the step-doubling method described in this paper to get a second-order accurate in time implicit finite difference scheme. BuGS also features step control for the convergence of Newton's method and automatic approximation of the Jacobi matrix.

For the spatial discretization, we used finite differences in space which correspond to continuous piecewise linear finite elements with mass lumping as the quadrature rule [13].

8.1. Problem. We consider the system, for $\beta(x, t), \rho(x, t)$, and $\alpha(x, t)$ :

$$
\begin{aligned}
\beta_{t}-\left(\beta \beta_{x}\right)_{x}-K\left(\beta \rho_{x}\right)_{x} & =0, & x \in[0, a], t \in\left[0, T_{f}\right], \\
\rho_{t}-\beta-K\left(\beta \rho_{x}\right)_{x}+\operatorname{rate}(\rho, \alpha) & =0, & x \in[0, a], t \in\left[0, T_{f}\right], \\
\alpha_{t}-\operatorname{rate}(\rho, \alpha) & =0, & x \in[0, a], t \in\left[0, T_{f}\right],
\end{aligned}
$$

where

$$
\operatorname{rate}(\rho, \alpha)=\max \left\{C \rho\left(\alpha_{\max }-\alpha\right), 0\right\}
$$

We take the boundary conditions

$$
\beta_{x}=\rho_{x}=0 \quad \text { on }\{0, a\}
$$

\footnotetext{
${ }^{1}$ Take $m$ positive and $v_{0}$ nonnegative. Suppose that for $1 \leq j \leq m, \Delta t_{j}$ is positive, $v_{j}, \alpha_{j}$, $\gamma_{j}$, and $\beta_{j}$ are nonnegative, and $\Delta t_{j} \beta_{j} \leq \frac{1}{2}$. Let $C_{m}=\exp \left(2.2 \sum_{j=1}^{m} \beta_{j} \Delta t_{j}\right)$. If, for each $j$, $\frac{v_{j}-v_{j-1}}{\Delta t_{j}}+\gamma_{j} \leq \alpha_{j}+\beta_{j}\left(v_{j}+v_{j-1}\right)$, then $v_{m}+\sum_{j=1}^{m} \gamma_{j} \Delta t_{j} \leq C_{m}\left\{v_{0}+\sum_{j=1}^{m} \alpha_{j} \Delta t_{j}\right\}$. Note that if $\beta_{j} \leq q$ for every $j$ and some constant $q$, then $C_{m} \leq \exp \left(2.2 q t_{m}\right)$.
} 


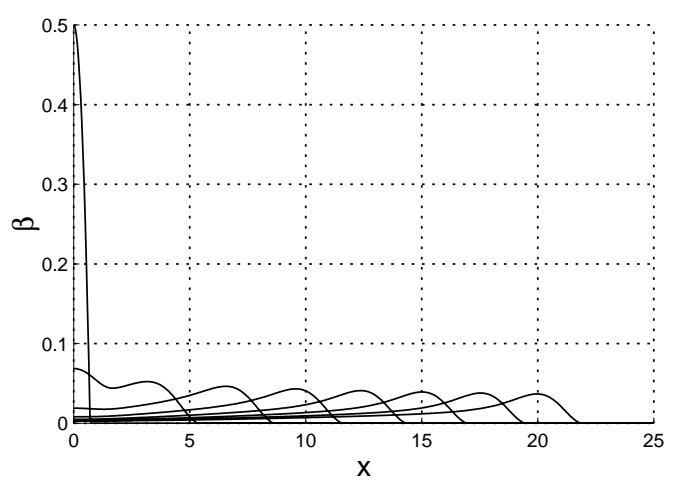

Figure 3. Bacterial population density profiles. Profiles are $t=2$ apart.

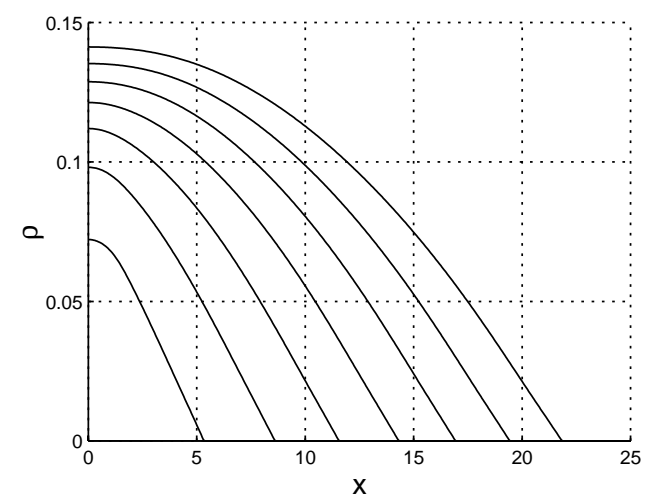

Figure 4. Repellent concentration profiles. Profiles are $t=2$ apart.

and the initial conditions

$$
\begin{aligned}
\beta(x, 0)=\max \left\{b-x^{2}, 0\right\}, & x \in[0, a], \\
\rho(x, 0)=0, & x \in[0, a], \\
\alpha(x, 0)=0, & x \in[0, a] .
\end{aligned}
$$

This system models the movement of bacteria on a petri dish. The bacteria are moving away from their own waste. The dependent variable $\beta$ is the bacterial population density, $\rho$ is the concentration of waste or repellent, and $\alpha$ is the amount of repellent absorbed by the laboratory medium.

8.2. Results. We consider the system of equations (8.1) -(8.6) with constants $K=$ $100, C=100, \alpha_{\max }=0.06, a=25, b=0.5, T_{f}=16$, and with $N=501$ uniform spatial nodes. BuGS gives Figures 36 as part of its output. Figures 3 [5 show the solutions as time profiles; each curve is the solution over space at a different point in time. The profiles are $\Delta t=2$ apart. Figure 6 shows the time steps taken during the solution of the system of partial differential equations. The oscillations in the time steps are due to the degenerate diffusion in the equations. When the sharp bacterial front enters a new space interval, step-doubling measures this as a more difficult problem and cuts the time step. 


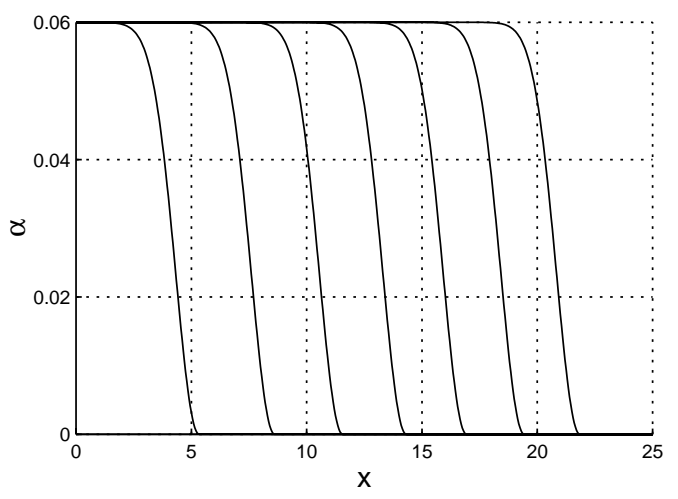

Figure 5. Absorbed repellent concentration profiles. Profiles are $t=2$ apart.

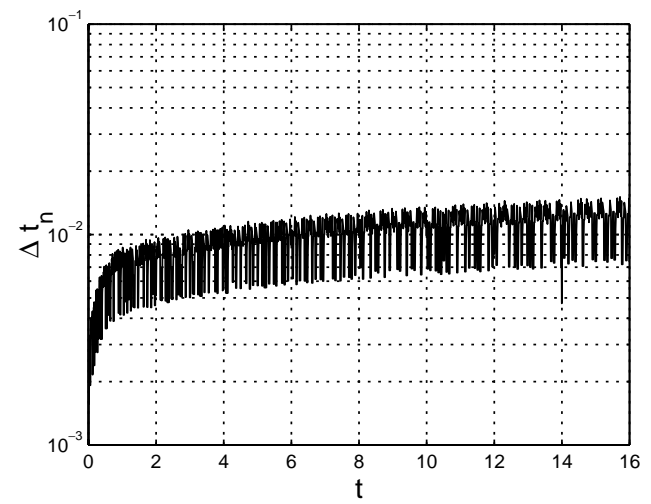

Figure 6. Step size for the calculation, measured $\log _{10}$. The oscillations are due to degeneracies in the parabolic system. When a new space interval is breached by the bacterial front, the error as measured by step-doubling increases.

For adaptivity in time, if $s$ is the solution from the single step and $d$ the solution from the two half-steps, then we required $\|d-s\|_{\infty}<0.001$ as the criterion for accepting a step. For other computational details, see the BuGS User Guide [1].

\section{Summary}

In this paper we discussed the use of step-doubling for time integration of parabolic partial differential equations. We presented one way to implement the stepdoubling algorithm that we have found particularly effective, and then compared step-doubling with Crank-Nicolson and theta-weighted extrapolation methods. The core of the paper consisted of a convergence analysis that was sufficiently general to allow for variable time steps, a useful result given that step-doubling is meant to be used as an adaptive method. One corollary of the convergence theorem is that the step-doubling Galerkin method is order $h^{2}+(\Delta t)^{2}$, where $h$ is the spatial mesh size, when the approximation space in space is the space of continuous piecewise linear 
functions. A spatial discretization using center finite differences corresponds to the use of continuous piecewise linear functions with mass lumping as the quadrature rule. We presented an example system whose numerical solution demonstrates the use of step-doubling for adaptive time-stepping, as well as its extension to nonlinear problems.

\section{REFERENCES}

1. Bruce P. Ayati, BuGS 1.0 user guide, Tech. Report CS-96-18, University of Chicago, 1996.

2. Bruce P. Ayati and Todd F. Dupont, Convergence of a step-doubling Galerkin method for parabolic problems, Tech. Report CS-99-02, University of Chicago, 1999.

3. James H. Bramble and Peter H. Sammon, Efficient higher order single step methods for parabolic problems. I, Math. Comp. 35 (1980), 655-677. MR.81h:65110

4. M. P. Brenner, X. D. Shi, and S. R. Nagel, Iterated instabilities during droplet fission, Phys. Rev. Letters 73 (1994), no. 25, 3391-3394.

5. Todd F. Dupont and A. E. Hosoi, Modeling and computation for applications in science and engineering, ch. Some reduced-dimension models based on numerical methods, pp. 59-80, Oxford University Press, 1998. MR2000i:76048

6. Jens Eggers, Nonlinear dynamics and breakup of free-surface flows, Rev. of Modern Phys. 69 (1997), no. 3, 865-929.

7. Jens Eggers and Todd F. Dupont, Drop formation in a one-dimensional approximation of the Navier-Stokes equation, J. Fluid Mech. 262 (1994), 205-221. MR94m:76029

8. C. William Gear, Numerical initial value problems in ordinary differential equations, Prentice-Hall, New Jersey, 1971. MR 47:4447

9. Gene H. Golub and Charles F. Van Loan, Matrix computations, third ed., The Johns Hopkins University Press, Baltimore, 1996. MR.97g:65006

10. A. E. Hosoi and John W. M. Bush, Evaporative instabilities in climbing films, J. Fluid Mech. 442 (2001), 217-239. MR

11. A. E. Hosoi and L. Mahadevan, Axial instability of a free-surface in a partially filled horizontal rotating cylinder, Physics of Fluids 11 (1999), 97-106. MR 99k:76055

12. L. F. Shampine, Local error estimation by doubling, Computing 34 (1985), 179-190. MR 87b:65093

13. Vidar Thomée, Galerkin finite element methods for parabolic problems, Springer Series in Computational Mathematics, vol. 25, Springer-Verlag, Berlin, 1997. MR.98m:65007

14. Mary F. Wheeler, A priori $L_{2}$ error estimates for Galerkin approximations to parabolic partial differential equations, SIAM J. Numer. Anal. 10 (1973), 723-759. MR.50:3613

Department of Mathematics, Southern Methodist University, Dallas, Texas 75275

E-mail address: ayati@smu.edu

Departments of Computer Science and Mathematics, The University of Chicago, Chicago, Illinois 60637

E-mail address: dupont@cs.uchicago.edu 